\title{
Um Kit de Ferramentas de Gestão do Conhecimento orientado à Inovação
}

\author{
Amaro Virginio da Silva Neto - amarovn@gmail.com ${ }^{1}$ \\ Ricardo André Cavalcante de Souza - ricardo.souza@ufrpe.br ${ }^{1}$
}

Resumo - Inovação é o meio pelo qual uma organização se diferencia em relação ao mercado. Um dos determinantes internos de inovação em uma organização é a gestão do conhecimento. A gestão do conhecimento considera o conhecimento um ativo que pode ser adquirido, recuperado e compartilhado em prol da melhoria dos processos de construção de produtos e serviços. Este trabalho apresenta um kit de ferramentas de gestão de conhecimento alinhado a critérios de inovação, o qual pode ser aplicado no contexto de processos de desenvolvimento de produtos de software.

Palavras-chave: Gestão do Conhecimento; Inovação; Práticas e Técnicas.

\section{An Innovation-driven Knowledge Management Toolkit}

Abstract - Innovation is a way for organizations to differentiate themselves from their competitors. One of the internal determinants of innovation in an organization is knowledge management. Knowledge Management considers knowledge an asset that can be acquired, recovered and shared in order to improve processes for construction of products and services. This paper presents a knowledge management toolkit aligned with innovation criteria which can be applied in the context of development processes of software product.

Keywords: Knowledge Management; Innovation; Practices and Techniques. 


\section{INTRODUÇÃO}

Para uma companhia ter sucesso ela necessita promover inovação (GORSCHEK et al., 20I0). Inovação refere-se a novas aplicações de conhecimentos, ideias, métodos e habilidades que podem gerar capacidades únicas e alavancar a competitividade de uma organização (ANDERSSON et al., 2008). Gestores identificam inovação insuficiente como um problema crucial para as organizações, porém não é fácil implementar com sucesso boas práticas de gestão da inovação (GASSMANN et al., 2006). O processo de gestão da inovação envolve a busca, seleção e implementação de ideias, bem como o aprendizado da equipe a partir do próprio processo de inovação (MOE et al., 20I2).

Segundo Moe (20I2), um dos determinantes internos para a inovação é a Gestão do Conhecimento (KM do inglês Knowledge Management). O principal objetivo da gestão de conhecimento é controlar e converter conhecimento tácito em explícito, facilitar o acesso e manter um gerenciamento integrado sobre as informações em seus diversos meios (DARRELL, 2009). O conhecimento tácito é pessoal, subjetivo e específico ao contexto. Já o conhecimento explícito é sistemático, objetivo e transmissível formalmente.

O processo SECI é utilizado com referência para a conversão entre os conhecimentos tácito e explícito (TAKEUCHI; NONAKA, 2008). O processo SECI consiste em quatro modos de conversão do conhecimento: Socialização, para a conversão de conhecimento tácito em conhecimento tácito, tal como a troca de conhecimento entre pessoas; Externalização, para conversão de conhecimento tácito em conhecimento explícito, tal como a escrita de um programa de software; Combinação, para conversão de conhecimento explícito em conhecimento explícito, tal como várias informações sintetizadas em um gráfico; e Internalização, para a conversão de conhecimento explícito em conhecimento tácito, tal como quando uma pessoa consegue aplicar continuamente um conhecimento adquirido em um manual de instruções.

Segundo Ichijo e Nonaka (2006), o desenvolvimento de um produto inicia com a Socialização, na qual o conhecimento tácito dos clientes é acumulado e compartilhado. Tal conhecimento tácito é então articulado em um conceito de produto através da Externalização. $\mathrm{O}$ conceito de produto é então sistematizado e transformado em um produto através da Combinação, na qual o conhecimento explícito coletado dentro e/ou fora da organização é selecionado, combinado e processado para formar conjuntos mais complexos e sistemáticos de conhecimento explícito. O conhecimento criado na forma de um novo produto é finalmente convertido em conhecimento tácito pelos clientes de mercado através da Internalização.

$\mathrm{Na}$ economia baseada em conhecimento, a indústria de software esta cada vez mais fortalecida, pois o produto final (software) serve como meio de automatização das tarefas e processos dos mais diversificados negócios. Porém, a engenharia de software, preocupada com o ciclo de vida, evolução e qualidade do produto, dá pouca ênfase na gestão do conhecimento durante o desenvolvimento do software.

Diante deste cenário, no qual a gestão do conhecimento é um dos determinantes de inovação das organizações, bem como necessita ser incorporado na engenharia de software, 
este artigo tem como objetivo apresentar um conjunto de ferramentas de gestão do conhecimento, resultante de um levantamento na literatura especializada, o qual pode ser usado por times de desenvolvimento de software para melhoria da comunicação, reuso e troca de conhecimentos, entre outros, de modo a melhorar o desempenho do processo produtivo.

Além da introdução, este trabalho está organizado em mais três seções. A Seção 2 apresenta o modelo de sustentação que orienta o desenvolvimento do trabalho. A Seção 3 apresenta o método de pesquisa que levou às contribuições do trabalho. A Seção 4 apresenta as considerações finais e propostas de trabalhos futuros.

\section{MODELO DE SUSTENTAÇÃO DO TRABALHO}

Este trabalho é dirigido por um modelo de sustentação descrito através da ferramenta Business Model Canvas (BMC) (OSTERWALDER; PIGNEUR, 20II). Este modelo de sustentação permite sintetizar: (a) contribuições da pesquisa; (b) quem irá se beneficiar com o resultado da pesquisa; (c) como a pesquisa foi conduzida; e (d) como o produto resultante da pesquisa se tornará sustentável. O Quadro I apresenta os blocos de construção preenchidos da ferramenta BMC com a estrutura do modelo de sustentação do trabalho.

Quadro I - BMC do Trabalho. Fonte: próprio Autor

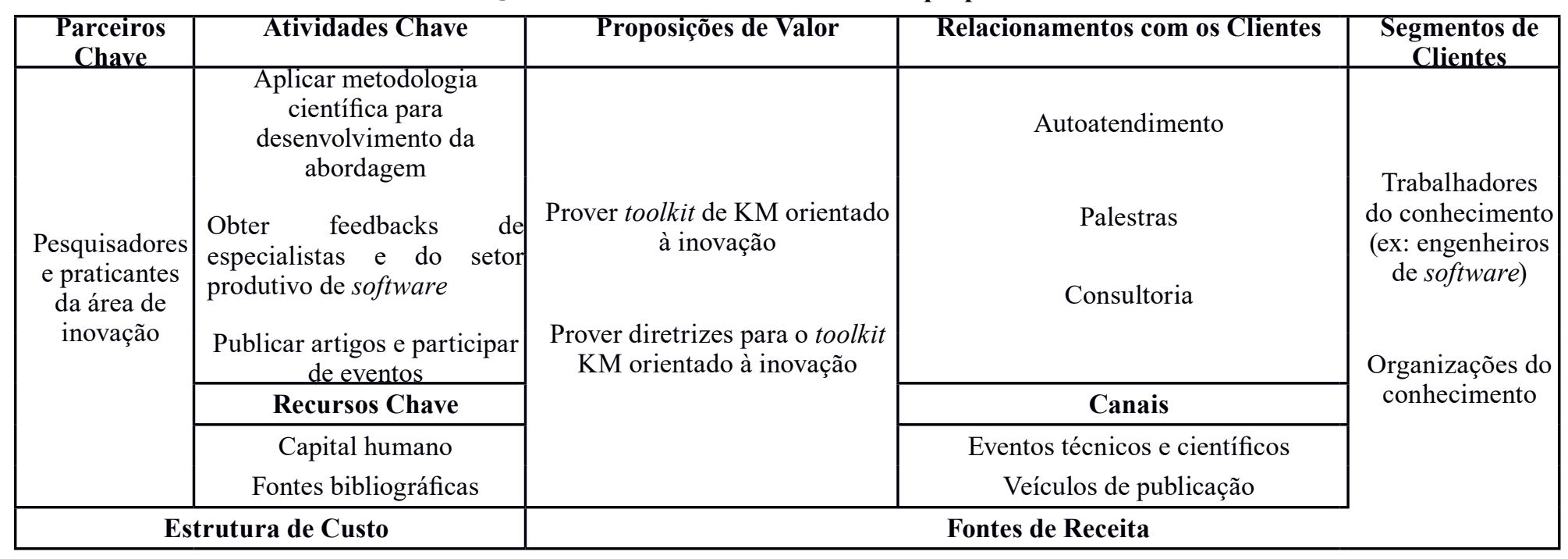

99 


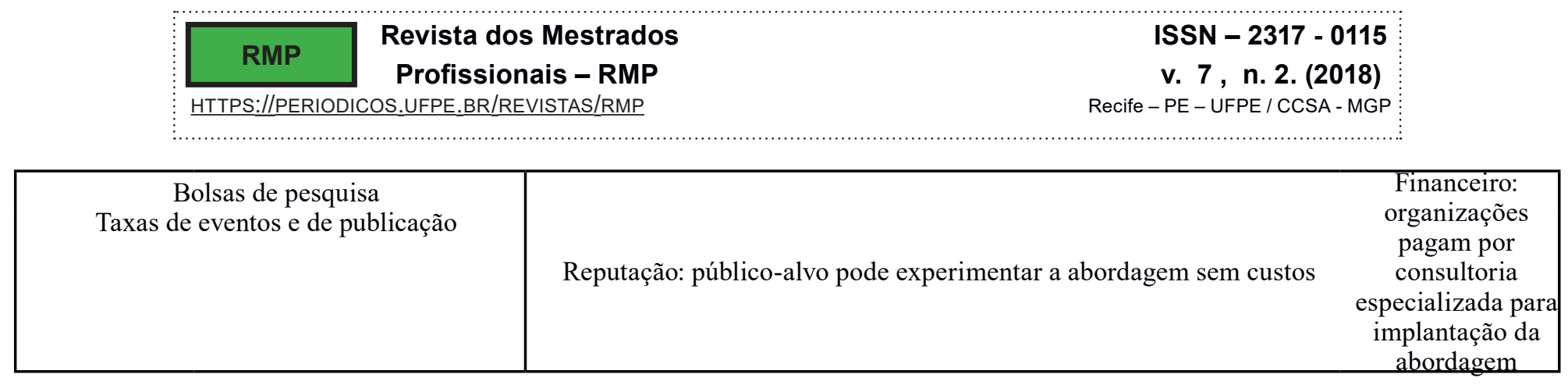

A estrutura do modelo de sustentação do trabalho apresentado no Quadro I descreve quais valores (Proposições de Valor) são entregues ao público-alvo (Segmentos de Clientes). Um valor é algo útil e observável que pode, por exemplo, ser usado para resolver um problema ou para auxiliar na execução de uma atividade. Os Canais são os meios pelos quais os clientes são alcançados visando à entrega dos valores propostos. Os Relacionamentos com os Clientes são as formas de interação com os clientes e como eles acessam o valor como, por exemplo, autoatendimento é quando o próprio cliente utiliza um valor sem a necessidade de auxílio do fornecedor.

As Fontes de Receita descrevem qual será o retorno do investimento (ROI do inglês Return of Investment) e como o negócio se tornará sustentável. O ROI pode ser direto, em forma de recursos financeiros, ou indiretos, como boa reputação ou status social. As Atividades Chave descrevem as principais ações necessárias para que o negócio (pesquisa aplicada) opere conforme planejado. Os Recursos Chave representam os principais ativos (físicos, intelectuais, lógicos, etc.) necessários para execução das atividades. Os Parceiros Chave representam a rede de fornecedores, pessoas e organizações que contribuem com o negócio (pesquisa). Por fim, a Estrutura de Custo representa as principais fontes de despesa para o negócio.

\section{MÉTODO DE PESQUISA}

Conhecimento é uma forma para a organização manter-se competitiva de maneira sustentável (BECKETT et al., 2000). O conhecimento gerado durante o processo de desenvolvimento é um ativo de valor para a companhia de software e por isso precisa ser capturado, armazenado, resgatado e reutilizado (TUAN; TUAN, 2011). No contexto da inovação, os projetos para construção de produtos ou serviços inovadores precisam de novos conhecimentos (TAVARES; PESSOA, 2014). Quanto mais radical é a inovação, maior é a necessidade de se obter ou utilizar um novo conhecimento (HALL; ANDRIANI, 2003).

A maior parte do conhecimento das pequenas e médias empresas de desenvolvimento de software está na forma tácita (experiência pessoal) e a transferência de conhecimento é algo que não está presente na cultura dessas empresas (NEVES et al., 20I4). O conhecimento é necessário para 
dar suporte ao processo de software, pois inclui uma visão geral do processo usado, descrição de tarefas para serem feitas, artefatos para serem produzidos e métodos para serem usados (HAVLICE; KUNSTAR, 2009).

Tendo em vista que o conhecimento é um dos principais ativos para uma organização, faz-se necessária a adoção de uma abordagem de gestão do conhecimento. Entre os benefícios da gestão do conhecimento estão: tempo de treinamento reduzido para os novos funcionários; agilidade na tomada de decisão; e maior eficiência operacional (CORBIN et al., 2007).

A Figura I apresenta em notação Business Process Model and Notation (BPMN) o método de pesquisa que orientou o desenvolvimento do trabalho. O trabalho de pesquisa iniciou com uma Revisão Bibliográfica, na qual foram consultadas obras referentes à gestão do conhecimento como determinante para a inovação nas organizações. A partir do resultado obtido na Revisão Bibliográfica, foi possível realizar as demais atividades do método de pesquisa, a serem detalhadas nas próximas subseções. Em cada produto de trabalho das atividades, descrito através de quadro, é apresentada a fonte de referência encontrada na Revisão Bibliográfica.

Figura I - Método de pesquisa do trabalho. Fonte: próprio Autor

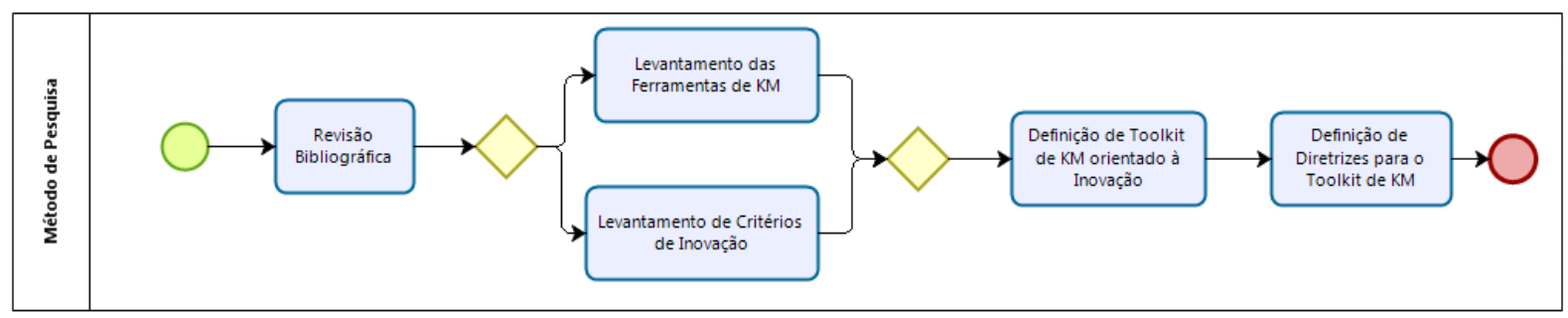

\subsection{Levantamento das ferramentas de KM}

A Gestão do Conhecimento (KM) é executada por meio de diversas práticas e técnicas e também com o auxílio de ferramentas de software. Para a implantação de uma cultura de gestão do conhecimento faz-se necessário identificar os mecanismos mais apropriados para uma organização. O Quadro 2 apresenta algumas das principais ferramentas de KM encontradas na literatura especializada.

\subsection{Levantamento de Critérios de Inovação}

A Gestão do Conhecimento deve contribuir para a busca da inovação na organização, propiciando uma abordagem estruturada visando o desenvolvimento sistemático de seus componentes de conhecimento tácito e explícito. Segundo Moe et al. (20I2), os aspectos de gestão do conhecimento que são determinantes internos para inovação em uma organização são:

- Compartilhamento de Conhecimento - o compartilhamento de conhecimento em conjunto com as capacidades inovadoras da organização são condições necessárias para desenvolvimentos futuros dentro da organização (ZHU et al., 2007). Negligenciar a importância das capacidades inovadoras na partilha de conhecimento pode levar a perda 
de desempenho. A organização deve então implementar uma cultura de compartilhamento de conhecimento, fortalecendo sua capacidade de inovação através da eficiência e aprendizagem organizacional;

Quadro 2 - Ferramentas de Gestão do Conhecimento. Fonte: compilado no interior do quadro

\begin{tabular}{|c|c|c|}
\hline $\begin{array}{l}\text { Ferramenta de } \\
\text { KM }\end{array}$ & Descriçãa & Fonte \\
\hline $\begin{array}{l}\text { Socialização } \\
\text { Organizacional }\end{array}$ & $\begin{array}{l}\text { Método através do qual os recém-chegados aprendem: } \\
\text { desempenhar suas funções; integrar-se à organização; e } \\
\text { adquirir conhecimentos e habilidades sociais necessárias } \\
\text { para o trabalho. }\end{array}$ & (WANG; ZHU, 2009) \\
\hline $\begin{array}{l}\text { Comunidades de } \\
\text { Práticas }\end{array}$ & $\begin{array}{l}\text { Grupo de pessoas que promovem a partilha de conhecimento } \\
\text { entre si. Este grupo de pessoas faz intercâmbio de } \\
\text { conhecimento e troca experiências sobre algum domínio } \\
\text { específico. }\end{array}$ & (ABDULLAH et al., 2014) \\
\hline $\begin{array}{l}\text { Sistema de } \\
\text { Recompensas }\end{array}$ & $\begin{array}{l}\text { Sistema de bônus e premiação usado para a motivação } \\
\text { pessoal de compartilhamento e retenção do conhecimento } \\
\text { na organização. }\end{array}$ & (ZHUGE, 2008) \\
\hline Feedback & $\begin{array}{l}\text { Retorno quanto ao uso do conhecimento para ajuste do } \\
\text { comportamento no processo de desenvolvimento. }\end{array}$ & $\begin{array}{l}\text { (RICHARDS; SCHIFFEL, } \\
2005)\end{array}$ \\
\hline $\begin{array}{l}\text { Fóruns de } \\
\text { Colaboração }\end{array}$ & $\begin{array}{l}\text { Sistema no qual usuários podem compartilhar informação, } \\
\text { aprender colaborativamente, e trocar experiências. }\end{array}$ & (SANI et al., 2013) \\
\hline Benchmarking & $\begin{array}{l}\text { Processo que avalia o desempenho de um objeto em relação } \\
\text { a outro. Usada para avaliar quão eficaz é a aplicação das } \\
\text { práticas de KM no processo de desenvolvimento. }\end{array}$ & (DENG, 2006) \\
\hline Protótipos & $\begin{array}{l}\text { Criação rápida de produto com funções básicas para obter } \\
\text { mais conhecimento sobre um problema. }\end{array}$ & (VIANNA et al., 2013) \\
\hline $\begin{array}{l}\text { Mapas do } \\
\text { Conhecimento }\end{array}$ & $\begin{array}{l}\text { Representações gráficas sobre: ativos de conhecimento, } \\
\text { fontes de conhecimento, estruturas de conhecimento, e } \\
\text { aplicações de conhecimento. }\end{array}$ & (PROBST et al., 2002) \\
\hline Reuniões & $\begin{array}{l}\text { Eventos que servem para anúncios ou para resolver } \\
\text { problemas que requeiram uma abordagem colaborativa. }\end{array}$ & (HAN; LUCZAJ, 2005) \\
\hline Livro de Bordo & $\begin{array}{l}\text { Registrar ideias de usuários ou membros do time durante o } \\
\text { desenvolvimento do produto. }\end{array}$ & (MALIK, 2014) \\
\hline $\begin{array}{l}\text { Sistematização do } \\
\text { Conhecimento }\end{array}$ & $\begin{array}{l}\text { Usado para sistematizar o conhecimento através de } \\
\text { ferramentas online, manuais, patentes, etc. }\end{array}$ & \begin{tabular}{|l|} 
(PERRY, 2003) \\
\end{tabular} \\
\hline $\begin{array}{l}\text { Acesso } \\
\text { Centralizado ao } \\
\text { Conhecimento }\end{array}$ & $\begin{array}{l}\text { Usado para disponibilizar o conhecimento de forma } \\
\text { centralizada na organização (por exemplo, através de } \\
\text { computação em nuvem). }\end{array}$ & (BOHLOULI et al., 2011) \\
\hline Experiências & $\begin{array}{l}\text { Forma de externalizar lições aprendidas para que os erros } \\
\text { não se repitam no futuro. }\end{array}$ & (DESOUZA, 2005) \\
\hline $\begin{array}{l}\text { Gestão do } \\
\text { conhecimento dos } \\
\text { stakeholders }\end{array}$ & $\begin{array}{l}\text { Sistema para obter e registrar conhecimento de fornecedores, } \\
\text { clientes e parceiros. }\end{array}$ & (COSTA; MEIRA, 2012) \\
\hline $\begin{array}{l}\text { Base de } \\
\text { Conhecimento }\end{array}$ & $\begin{array}{l}\text { Armazenar conhecimentos adquiridos nos processos de } \\
\text { desenvolvimento de produtos da organização. }\end{array}$ & (PEDELTY, 1989) \\
\hline Mentoring & $\begin{array}{l}\text { Pratica para transferência de conhecimento, de forma } \\
\text { pessoal ou profissional. Um mentor treina outra pessoa } \\
\text { menos experiente. }\end{array}$ & (DESOUZA, 2005) \\
\hline Wiki & $\begin{array}{l}\text { Repositório que pode ser editado por qualquer pessoa. As } \\
\text { mudanças de conteúdo são registradas e disponibilizadas } \\
\text { para a comunidade. }\end{array}$ & (MONTICOLO et al., 2012) \\
\hline
\end{tabular}

- Habilidades de Aprendizado Organizacional - correspondem a como os empregados compartilham conhecimento, especialmente sobre aspectos relacionados aos clientes (AIMAN-SMITH et al., 2005). Isto ajuda a criar um conhecimento profundo sobre a Inovação de Valor (conceito onde todos os empregados podem inovar e criar valor). Como resultado a organização está hábil para crescer e continuar se adaptando a mudanças no ambiente; 
- Capital Organizacional - conhecimento institucionalizado dentro de processos, base de dados, patentes e manuais que as organizações usam para reter o conhecimento (CARMONA-LAVADO et al., 20Io). Criar um capital organizacional requer informações e habilidades adquiridas a partir das atividades de inovação;

- Variedade de Fontes de Conhecimento - é um determinante de inovação por que independentemente do tamanho, as organizações não conseguem criar todo o conhecimento que contribuem para o desenvolvimento inovador de sucesso (AMARA et al., 2009). As organizações precisam de uma grande variedade de fontes externas e complementares de conhecimento;

- Difusão do Conhecimento - serve para fortalecer a capacidade de inovação de uma entidade através da análise e aproveitamento dos conhecimentos interno e externo (TSENG, 2009). O resultado da inovação da organização depende do contexto em que ela está inserida (clientes, mercado e localização geográfica);

- $\quad$ Treinamento e Educação da Equipe - estão associados ao sucesso de projetos inovadores (GEBAUER et al., 2008). Uma equipe que possui o domínio de uma atividade e que está bem informada tem mais chance de fornecer produtos adequados ao cliente. Uma equipe bem-educada também consegue identificar mais facilmente oportunidades de inovação, e proporciona ao cliente produtos personalizados e com alto valor agregado;

- Geração de Ideia - A inovação começa a partir de boas ideias, ou seja, a Geração de Ideias é uma importante variável para a capacidade de inovação das organizações (KOC, 2007). A geração de ideia é uma forma individual e organizacional de coletar, disseminar e usar conhecimento obtido através de: grupos de pesquisas dedicados, experimentação, experiência da equipe e sistematização da gestão do conhecimento.

O Quadro 3 sintetiza os critérios de inovação relacionados à Gestão do Conhecimento.

Quadro 3 - Critérios de Inovação. Fonte: baseado em Moe et al. (20I2)

\begin{tabular}{|c|c|c|}
\hline Identificador & Critério & Descrição \\
\hline $\mathrm{C} 01$ & $\begin{array}{l}\text { Compartilhamento de } \\
\text { Conhecimento }\end{array}$ & $\begin{array}{l}\text { Implantação de uma cultura de compartilhamento de conhecimento } \\
\text { dentro da organização. }\end{array}$ \\
\hline $\mathrm{C} 02$ & $\begin{array}{l}\text { Habilidades de } \\
\text { Aprendizagem } \\
\text { Organizacional }\end{array}$ & $\begin{array}{l}\text { Como os empregados socializam conhecimento entre si na } \\
\text { organização. }\end{array}$ \\
\hline $\mathrm{C} 03$ & Capital Organizacional & $\begin{array}{l}\text { Ativos de conhecimento institucionalizados dentro de processos, } \\
\text { base de dados, patentes e manuais. }\end{array}$ \\
\hline $\mathrm{C} 04$ & $\begin{array}{l}\text { Variedade de Fontes de } \\
\text { Conhecimento }\end{array}$ & Fontes internas e externas de conhecimento da organização. \\
\hline $\mathrm{C} 05$ & $\begin{array}{l}\text { Difusão de } \\
\text { Conhecimento }\end{array}$ & Análise da eficiência do conhecimento no processo de inovação. \\
\hline $\mathrm{C} 06$ & $\begin{array}{l}\text { Formação e Educação } \\
\text { das Pessoas }\end{array}$ & $\begin{array}{l}\text { Treinar a expertise e capacitações das pessoas para identificar } \\
\text { oportunidades de inovação. }\end{array}$ \\
\hline $\mathrm{C} 07$ & Geração de ideias & $\begin{array}{l}\text { Base da inovação. Ideias resultantes do trabalho colaborativo e da } \\
\text { sistematização do processo. }\end{array}$ \\
\hline
\end{tabular}

\subsection{Definição de toolkit de KM orientado à inovação}

A primeira proposição de valor especificada no Modelo de Sustentação do Trabalho 
(Quadro I) é "prover toolkit de KM orientado à inovação". O Quadro 4 sintetiza a primeira proposição de valor prometida por este trabalho. Para tanto, foram identificadas quais ferramentas de KM (Quadro 2) satisfazem cada critério determinante para a inovação (Quadro 3).

Quadro 4 - Toolkit de KM orientado à inovação. Fonte: próprio Autor

\begin{tabular}{|c|c|c|}
\hline $\begin{array}{l}\text { Critério de } \\
\text { Inovação }\end{array}$ & Propósito no contexto de inovação & Toolkit de KM \\
\hline $\mathrm{C} 01$ & $\begin{array}{l}\text { Incentivar a cultura do } \\
\text { compartilhamento de conhecimento. }\end{array}$ & $\begin{array}{l}\text { - } \text { Sistema de Recompensa } \\
\text { - } \text { Comunidades de Prática } \\
\text { - Socialização Organizacional } \\
\text { - } \quad \text { Relatos de Experiências }\end{array}$ \\
\hline $\mathrm{C} 02$ & $\begin{array}{l}\text { Garantir que o conhecimento seja } \\
\text { compartilhado na organização. }\end{array}$ & $\begin{array}{ll}\text { - } & \text { Socialização de Pessoas } \\
\text { - } & \text { Comunidades de Práticas } \\
\text { - } & \text { Fóruns de Colaboração } \\
\text { - } & \text { Wiki } \\
\text { - } & \text { Reuniões } \\
\text { - } & \text { Relatos de Experiências }\end{array}$ \\
\hline $\mathrm{C} 03$ & $\begin{array}{l}\text { Institucionalizar o conhecimento dentro } \\
\text { da organização. }\end{array}$ & $\begin{array}{ll}\text { - } & \text { Sistematização do Conhecimento } \\
\text { - } & \text { Base de Conhecimento } \\
\text { - } & \text { Wiki } \\
\text { - } & \text { Livro de Bordo } \\
\text { - } & \text { Acesso centralizado ao conhecimento }\end{array}$ \\
\hline $\mathrm{C} 04$ & $\begin{array}{l}\text { Gerenciar fontes de conhecimento dentro } \\
\text { e fora da organização. }\end{array}$ & $\begin{array}{l}\text { - } \text { Gerenciamento do Conhecimento das Partes } \\
\text { - } \quad \text { Mapa de Conhecimento } \\
\text { - } \quad \text { Base de Conhecimentos } \\
\end{array}$ \\
\hline $\mathrm{C} 05$ & $\begin{array}{l}\text { Analisar a eficácia da aplicação do } \\
\text { conhecimento no processo de inovação. }\end{array}$ & $\begin{array}{l}\text { - } \quad \text { Benchmarking } \\
\text { - } \quad \text { Feedback }\end{array}$ \\
\hline $\mathrm{C} 06$ & $\begin{array}{l}\text { Garantir conhecimento adequado a todos } \\
\text { os membros da organização. }\end{array}$ & $\begin{array}{ll}\text { - } & \text { Comunidades de Práticas } \\
\text { - } & \text { Mentoring } \\
\end{array}$ \\
\hline $\mathrm{C} 07$ & $\begin{array}{l}\text { Garantir a geração de inovação a partir } \\
\text { de novas ideias. }\end{array}$ & $\begin{array}{ll}\text { - } & \text { Prototipagem } \\
\text { - } & \text { Brainstorming } \\
\end{array}$ \\
\hline
\end{tabular}

\subsection{Diretrizes para Práticas e Técnicas de KM}

A segunda proposição de valor especificada no Modelo de Sustentação do Trabalho (Quadro I) é "prover diretrizes para o toolkit de KM orientado à inovação". No contexto deste trabalho, diretrizes são boas práticas, encontradas na literatura especializada, para direcionar a execução das ferramentas de gestão do conhecimento.

O Quadro 5 sintetiza as principais diretrizes para o toolkit de KM orientado à inovação (Quadro 4), bem como as respectivas fontes de referência de tais diretrizes. 
Quadro 5 - Diretrizes para o toolkit de KM orientado à inovação. Fonte: compilado no interior do quadro

\begin{tabular}{|c|c|c|}
\hline Toolkit de KM & Diretrizes & Fonte \\
\hline $\begin{array}{l}\text { Sistema de } \\
\text { Recompensa }\end{array}$ & $\begin{array}{ll}\text { - } & \text { Valorizar trabalhos realizados pelo time } \\
\text { - } & \text { Manter uma equidade das recompensas } \\
\text { - } & \text { Alinhar trabalhos com as metas da organização }\end{array}$ & (BEI, 2013) \\
\hline $\begin{array}{l}\text { Socialização } \\
\text { Organizacional }\end{array}$ & $\begin{array}{l}\text { - Compartilhar a história, a linguagem e as políticas da } \\
\text { organização } \\
\text { - Ter um manual de conduta das pessoas } \\
\text { - Definir Metas e valores } \\
\text { - Incentivar a proficiência }\end{array}$ & (CHAO et al., 1994) \\
\hline $\begin{array}{l}\text { Comunidades de } \\
\text { Práticas }\end{array}$ & $\begin{array}{ll}\text { - } & \text { Construir comunidades em torno de poucos e relevantes } \\
\text { assuntos } \\
\text { - Desenvolver líderes das comunidades } \\
\text { - Construir rede de comunicação } \\
\text { - Dar suporte adequado às comunidades de Práticas }\end{array}$ & (KAEHKOENEN, 2004) \\
\hline $\begin{array}{l}\text { Fóruns de } \\
\text { Colaboração }\end{array}$ & $\begin{array}{ll}\text { - } & \text { Analisar as questões e respostas } \\
\text { - } & \text { Pontuar, sumarizar e combinar as sentenças }\end{array}$ & (SANI et al., 2013) \\
\hline Wiki & 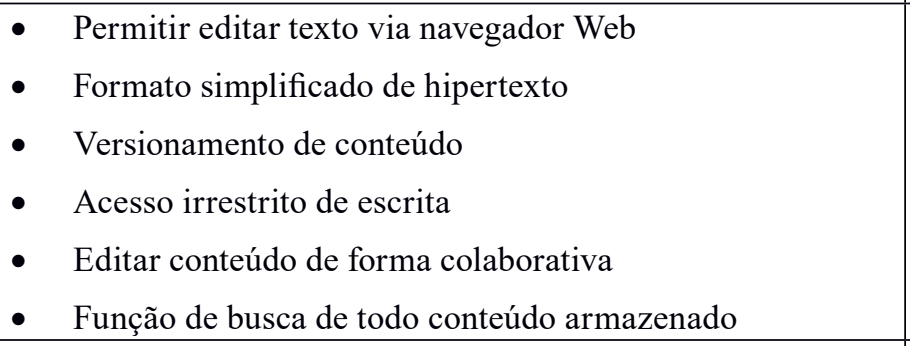 & $\begin{array}{l}\text { (MONTICOLO et al., } \\
\text { 2012) }\end{array}$ \\
\hline $\begin{array}{l}\text { Sistematização do } \\
\text { Conhecimento }\end{array}$ & $\begin{array}{l}\text { - Classificar o conhecimento } \\
\text { - Codificar o conhecimento tácito } \\
\text { - Organizar instâncias do conhecimento } \\
\text { - Codificar conhecimento de forma apropriada para ser } \\
\text { armazenado } \\
\text { - Reusar e compartilhar conhecimento }\end{array}$ & (YOSHIKAWA, 1993) \\
\hline $\begin{array}{l}\text { Base de } \\
\text { Conhecimento }\end{array}$ & $\begin{array}{l}\text { - Definir e seguir uma estratégia gobal da Base de } \\
\text { Conhecimento para a organização; } \\
\text { - A construção de uma Base de Conhecimento deve ser } \\
\text { restrita aos recursos organizacionais } \\
\text { - Construir a Base de Conhecimento, após a seleção da } \\
\text { estratégia a análise da viabilidade } \\
\text { - Aplicar a Base de Conhecimento nos projetos } \\
\text { - Realizar ações necessárias para a manutenção de Base de } \\
\text { Conhecimento }\end{array}$ & (DUTTA, 1997) \\
\hline $\begin{array}{l}\text { Gerenciamento do } \\
\text { Conhecimento dos } \\
\text { Stakeholders }\end{array}$ & $\begin{array}{l}\text { - Desenhar um processo de negócio que pode melhorar a } \\
\text { satisfação do cliente } \\
\text { - Analisar a causa dos problemas dos clientes } \\
\text { - Definir metas de satisfação para as partes interessadas } \\
\text { - Compartilhar conhecimento com as partes interessadas }\end{array}$ & (JYU et al., 2009) \\
\hline
\end{tabular}




\begin{tabular}{|c|c|c|}
\hline Toolkit de KM & Diretrizes & Fonte \\
\hline $\begin{array}{l}\text { Mapa de } \\
\text { Conhecimento }\end{array}$ & $\begin{array}{l}\text { - Identificar etapas dos processos organizacionais sensíveis } \\
\text { ao conhecimento } \\
\text { - Localizar e indexar ativos de conhecimento } \\
\text { - Integrar os ativos indexados em um sistema de navegação } \\
\text { que facilite a busca pelo conhecimento } \\
\text { - Possibilitar mecanismos descentralizados de atualização } \\
\text { dos ativos de conhecimento }\end{array}$ & (PROBST et al., 2002) \\
\hline Benchmarking & $\begin{array}{l}\text { - } \\
\text { - Aefinir critérios de avaliação } \\
\text { - } \text { relativa dos critérios de avaliação } \\
\text { - Usar variáveis linguísticas para ponderar os critérios de } \\
\text { avaliação } \\
\text { - Calcular critérios de avaliação de desempenho } \\
\text { - Produzir uma matriz de desempenho } \\
\text { - Calcular o índice de desempenho global de cada critério de } \\
\text { avaliação }\end{array}$ & (DENG, 2006) \\
\hline Mentoring & $\begin{array}{l}\text { - } \\
\text { - } \\
\text { - } \\
\text { - Ensinarinar as "qualidades de um bom mentor" } \\
\text { - Experimentar novas técnicas e metodologias de ensino } \\
\text { - Prover suporte ao aluno } \\
\text { - } \\
\text { Profissionalismo }\end{array}$ & $\begin{array}{l}\text { (PARKER-KATZ; BAY, } \\
\text { 2008) }\end{array}$ \\
\hline Brainstorming & $\begin{array}{ll}\text { - } & \text { Analisar problemas } \\
\text { - } & \text { Propor soluções criativas para os problemas } \\
\text { - } & \text { Combinar e aprimorar ideias } \\
\text { - } & \text { Não pré-julgar ideias de outras pessoas } \\
\end{array}$ & (VIANNA et al., 2013) \\
\hline Protótipos & $\begin{array}{ll}\text { - } & \text { Formular questões sobre o problema a ser tratado } \\
\text { - } & \text { Criar, testar e avaliar protótipo(s)/experimento(s) }\end{array}$ & (VIANNA et al., 2013) \\
\hline
\end{tabular}

\section{Conclusão}

Este trabalho procurou prover para os trabalhadores do conhecimento (ex: engenheiros de software), que buscam a melhoria de processos (ex: desenvolvimento de software), um ferramental para fomentar a inovação a partir do uso de ferramentas de gestão do conhecimento.

Inicialmente, foi realizada uma pesquisa bibliográfica na qual foi possível identificar algumas das principais ferramentas de gestão do conhecimento de acordo com dezessete artigos especializados na área de gestão do conhecimento. As práticas e técnicas de gestão do conhecimento levantadas na pesquisa bibliográfica foram então analisadas e algumas delas se mostraram em conformidade com os critérios de inovação relacionados à gestão do conhecimento definidos por Moe et al. (20I2). Finalmente, para o conjunto de ferramentas de gestão do conhecimento (toolkit de KM) orientado à inovação, foram fornecidas diretrizes de acordo com recomendações de doze trabalhos encontrados na literatura especializada. 
Tendo em vista que a Gestão do Conhecimento (KM) é um determinante para inovação nas organizações, a contribuição deste trabalho consistiu no levantamento de um toolkit de KM orientado à inovação, ou seja, um conjunto de ferramentas de gestão do conhecimento com o propósito de atender critérios que podem levar à inovação, bem como diretrizes, isto é, boas práticas para uso de tais ferramentas do toolkit de KM. Este toolkit de KM é útil para equipes e organizações que pretendem utilizar os conhecimentos tácitos e explícitos internos e externos para melhorar o desempenho dos processos produtivos, como no caso de desenvolvimento de produtos de software, visando à inovação.

As propostas de trabalho futuro são: aplicar esse toolkit de KM orientado à inovação em um contexto de processos de desenvolvimento de produtos de software; e obter feedback de especialistas e do setor produtivo quanto as proposições de valor prometidas neste trabalho (Quadro I).

\section{Referência}

ABDULLAH, R.; ABDULLAH, S.; TEE, M. Web-Based Knowledge Management Model for Managing and Sharing Green Knowledge of Software Development in Community of Practice. 8th Malaysian Software Engineering Conference (MySEC), p. 2IO-215, 2014.

AIMAN-SMITH, L.; GOODRICH, N.; ROBERTS, D.; SCINTA, J. Assessing your organization's potential for value innovation. Research-Technology Management, v. 48, n. 2, p. 37-42, 2005.

AMARA, N.; LANDRY, R.; DOLOREUX, D. Patterns of innovation in knowledge-intensive business services. The Service Industries Journal, v. 29, n. 4, p. 407-430, 2009.

ANDERSSON, M.; LINDGREN, R.; HENFRIDSSON, O. Architectural knowledge in interorganizational IT Innovation. Journal of Strategic Information Systems, v. I7, p. 19-38, 2008.

BECKETT, A.; WAINWRIGHT, C.; BANCE, D. Knowledge management: strategy or software? Management Decision, v. 38, n. 9, p. 60I-606, 2000. Disponível em: <http://www. emeraldinsight.com/journals.htm?articleid $=865175 \&$ show $=$ abstract $>$. Acesso em: $15 / \mathrm{I} / 2015$.

BEI, J. L. The selection of incentive team-based reward model. International Conference on Management Science and Engineering - Annual Conference Proceedings, p. 987-992, 2013.

BOHLOULI, M.; HOLLAND, A.; FATHI, M. Knowledge integration of collaborative product design using cloud computing infrastructure. 20II Ieee International Conference on Electro/ Information Technology, p. I-8, 20II.

CARMONA-LAVADO, A.; CUEVAS-RODRÍGUEZ, G.; CABELLO-MEDINA, C. Social and organizational capital: Building the context for innovation. Industrial Marketing Management, v. 39, n. 4, p. 68I-69o, 2010. 
CHAO, G. T.; O’LEARY-KELlY, A. M.; WOLF, S.; KLEIN, H. J.; GARDNER, P. D. Organizational Socialization: Its Conten and Consequences. Journal of Applied Psychology, v. 79, n. 5 , p. $730-743$, I994.

CORBIN, R. D.; DUNBAR, C. B.; ZHU, Q. A three-tier knowledge management scheme for software engineering support and innovation. Journal of Systems and Software, v. 80, n. 9, p. I494-I505, 2007.

COSTA, R. A.; MEIRA, S. R. L. Analysis of a Social Network as a Knowledge Management Tool. Brazilian Symposium on Collaborative Systems (SBSC), p. I54-I62, 2012.

DARRELL, K. Innovation in Turbulent Times. Harvard Business Review, 2009.

DENG, H. A Decision Model for Benchmarking Knowledge Management Practices. 2006 International Conference on Computational Inteligence for Modelling Control and Automation and International Conference on Intelligent Agents Web Technologies and International Commerce (CIMCA'06), p. 233-233, 2006.

DESOUZA, K. C. Experiences with Conducting Project Postmortems : Reports vs . Stories and Practitioner Perspectives Experiences with Conducting Project Postmortems : Reports vs . Stories and Practitioner Perspectives Abstract. Review Literature And Arts Of The Americas, v. OO, n. C, p. I-20, 2005.

DUTTA, S. Strategies for Implementing Knowledge-Based Systems. IEEE TRANSACTIONS ON ENGINEERING MANAGEMENT, v. 44, n. I, p. 79-90, I997.

GASSMANN, O.; SANDMEIER, P.; WECHT, C. H. Extreme customer innovation in thr front-end: learning from a new software paradigm. International Journal of Technology Management, v. 33, p. 46-66, 2006.

GEBAUER, H.; KREMPL, R.; FLEISCH, E.; FRIEDLI, T. Innovation of product-related services. Managing Service Quality, v. I8, n. 4, p. 387-404, 2008.

GORSCHEK, T.; FRICKER, S.; PALM, K.; KUNNSMAN, S. A Lightweight Innovation Process for Sftware-Intensive Product Development. 27th ed. IEE Software, $20 I 0$.

HALL, R.; ANDRIANI, P. Managing knowledge associated with innovation. Journal of Business Research, v. 56, n. 2, p. I45-I52, 2003.

HAN, C. Y.; LUCZAJ, J. E. Collaborative Knowledge Management in e-Meetings University of Cincinnati Dept of Mathematics Computer Science College of Mount Saint Joseph. Collaborative Technologies and Systems, 2005. Proceedings of the 2005 International Symposium on, p. I52-I59, 2005.

HAVLICE, Z.; KUNSTAR, J. Knowledge in software life cycle. $7_{\text {th }}$ International Symposium on Applied Machine Intelligence and Informatics, p. I53-I57, 2009. 
ICHIJO, K.; NONAKA, I. Knowledge Creation and Management. USA: Oxford University Press, 2006.

JYU, J.; YANG, S. C.; CHEN, C. W. Transform customer knowledge into company valuecase of a global retailer. 6th International Conference on Service Systems and Service Management, p. 959-964, 2009.

KAEHKOENEN, T. Agile Methods for Large Organizations - Building Communities of Practice. Agile Development Conference, p. 2-IO, 2004.

KOC, T. Organizational determinants of innovation capacity in software companies. , v. 53, p. $373-385,2007$.

MALIK, M. Understanding the use of paper and online logbooks for final year undergraduate engineering projects. Frontiers in Education Conference (FIE), 2014 IEEE, p. I-4, $20 I 4$.

MOE, N.; BARNEY, S.; AURUM, A.; et al. Fostering and sustaining innovation in a fast growing company. International Conference on Product - Focused Software Development and Process Improvement (PROFES), 2012.

MONTICOLO, D.; MOREL, L.; BOLY, V. How a semantic Wiki can support the ideas development in innovation activities. International Conference on Computer and Information Science, ICCIS 20I2, v. I, p. 79-83, 2012.

NEVES, S. M.; SILVA, C. E. S. DA; SALOMON, V. A. P.; SILVA, A. F. DA; SOTOMONTE, B. E. P. Risk management in software projects through Knowledge Management techniques: Cases in Brazilian Incubated Technology-Based Firms. International Journal of Project Management, v. 32, n. I, p. I25-I38, 2014.

OSTERWALDER, A.; PIGNEUR, Y. Business Model Generation: Inovação em Modelos de Negócios. Rio de Janeiro: Alta Books, 20II.

PARKER-KATZ, M.; BAY, M. Conceptualizing mentor knowledge: Learning from the insiders. Teaching and Teacher Education, v. 24, n. 5, p. I259-I269, 2008.

PEDELTY, M. J. Command Sail: a prototype knowledge base system for a commander's associate. IEEE Energy and Information Technologies in the Southeast', p. I40-I45, I989.

PERRY, I. Making sense of the organisation's knowledge: does systematisation of the knowledge base have a positive or negative effect on organizational culture. 36th Annual Hawaii International Conference on System Sciences, 2003.

PROBST, G.; RAUB, S.; ROMHARDT, K. Gestão do Conhecimento Os elementos construtivos do sucesso. Porto Alegre: Bookman, 2002.

RICHARDS, M.; SCHIFFEL, J. A distance learning framework for automatic instructor replies: 
articulable tacit knowledge used for feedback upon request. Proceedings. IEEE SoutheastCon, 2005., p. 6II-620, 2005.

SANI, M. R. F.; KARDAN, A. A.; COHAN, A. A supporting tool in online learning forums based on multi-documents summarization. 4th International Conference on e-Learning and e-Teaching, ICELET 20I3, p. 30-35, 2013.

TAKEUCHI, H.; NONAKA, I. Gestão do Conhecimento. São Paulo: bookman, 2008.

TAVARES, E. DA S.; PESSOA, M. S. DE P. Projetos com inovação tecnológica: proposta de um modelo integrativo entre gerência de projetos e gerência do conhecimento numa perspectiva cliente-fornecedor., v. II, n. I, p. IO5-I30, 2014.

TSENG, C.-Y. Technological innovation and knowledge network in Asia: Evidence from comparison of information and communication technologies among six countries. Technological Forecasting and Social Change, v. 76, n. 5, p. 654-663, 2009.

TUAN, D. T.; TUAN, D. C. Enhance Java Software Development with Knowledge Acquisition and Management Tools. 2oII Third International Conference on Knowledge and Systems Engineering, 20II.

VIANNA, M.; VIANNA, Y.; ADLER, I. K.; LUCENA, B.; RUSSO, B. Design Thinking Inovação em Negócios. $2^{\mathrm{a}}$ ed. Rio de Janerio: MJV PRESS, 2013.

WANG, Y.; ZHU, Y. The relationships among organizaitonal socialization, trust, knowledge sharing and creative behavior. Proceedings - International Conference on Management and Service Science, MASS 2009,, n. 70702023, p. 3-7, 2009.

YOSHIKAWA, H. Systematization of Design Knowledge. Annals of the CIRP, v. 42, p. I3II34, I993.

ZHU, Y.; WANG, Y.; LAN, H. Innovative capabilities in the process of knowledge sharing to firm performance. 2007 International Conference on Wireless Communications, Networking and Mobile Computing, WiCOM 2007, p. 5394-5397, 2007.

ZHUGE, J. Reward systems for implementing knowledge sharing in knowledge - intensive corporation. Proceedings - ISECS International Colloquium on Computing, Communication, Control, and Management, CCCM 2008, v. I, p. 5I4-5I8, 2008. 